\title{
Revascularização do miocárdio sem circulação extracorpórea com derivação intraluminal temporária (DILT) na emergência pós angioplastia transluminal coronária (ATC)
}

\author{
Ana M. Rocha PINTO*, Marcelo POCHINI*, Itagiba MARINELLI*, Valquíria P. CAMPAGNUCCI*, \\ Sílvio M. A. GANDRA*, Luiz Antônio RIVETTI*
}

RBCCV $44205-301$

Pinto A M R, Pochini M, Marinelli I, Campagnucci V P, Gandra S M A, Rivetti L A - Revascularizaçăo do miocárdio sem circulaçāo extracorpórea com derivaçāo intraluminal temporária (DILT) na emergência pós angioplastia transluminal coronária (ATC). Rev Bras Cir Cardiovasc 1996;11 (3): 137-42.

RESUMO: No periodo de novembro 1989 a dezembro 1995, 18 doentes foram submetidos a cirurgia de emergéncia para revascularizaçăo do miocárdio sem circulaçăo extracorpórea (CEC), devido a insucesso de angioplastia transluminal percutânea (ATC). Todos os pacientes apresentavam lesōes do ramo interventricular anterior (RIA), coronária direita (CD) ou coronária diagonal $(\mathrm{Dg})$, passiveis de abordagem sem CEC. Em todos utilizamos derivaçāo intraluminal temporária (DITL) para manter o fluxo coronariano e minimizar a isquemia. Três $(16,66 \%)$ doentes foram operados na vigência de infarto agudo do miocárdio e $4(22,22 \%)$ doentes com importante corrente de lesāo ao ECG. O choque cardiogênico estava presente em $3(16,66 \%)$ doentes. Nāo houve mortalidade. Realizamos estudo estatístico para comparaçăo da mortalidade referida por diversos autores utilizando revascularizaçăo com CEC.

DESCRITORES: Revascularizaçăo do miocárdio, métodos. Revascularizaçăo do miocárdio, cirurgia. Angioplastia. Vasos coronários, cirurgia. Próteses vasculares.

\section{INTRODUÇĀO}

Desde sua introduçăo em 1978 (1) a angioplastia transiuminal coronária (ATC) vem sendo cada vez mais utilizada e vem se aprimorando. Os casos de necessidade de operaçăo de emergência devido a insucesso de ATC têm sido bem estudados na literatura $(2-7)$ apresentam, na atualidade, uma incidência entre $2,8 \%$ a $7 \%(6-9)$.
Devido à instabilidade hemodinâmica com que se apresentam estes doentes, alguns em choque cardiogênico, o risco cirúrgico é elevado, variando de $4,6 \%$ a $30 \%(2,6,9)$, apesar do emprego das novas técnicas de preservação do miocárdio.

A técnica de revascularização do miocárdio sem o uso da CEC tem sua história iniciada com CARREL (10), em 1910, que realizou uma derivação da aorta des-

Trabalho realizado na Faculdade de Ciâncias Médicas da Santa Casa de Misericórdia de São Paulo e no Hospital Samaritano. Såo Paulo, SP, Brasil. Apresentado ao $23^{\circ}$ Congresso Nacional de Cirurgia Cardiaca. Recife, PE, 20 a 23 de março, 1996.

"Da Faculdade de Ciências Médicas da Santa Casa de Misericórdia de Săo Paulo e do Hospital Samaritano.

Endereço para correspondéncia: Ana M. Rocha Pinto. Rua Itacolomi 576 - Apto 51. São Paulo, SP, Brasil. CEP: 01239-020. Tel. (01 1) 259-6943. 
Pinto A M R, Pochini M, Marinelli I, Campagnucci V P, Gandra S M A, Rivetti L A - Revascularizaçāo do miocárdio sem circulaçāo extracorpórea com derivaçāo intraluminal temporária (DILT) na emergência pós angioplastia transluminal coronária (ATC). Rev Bras Cir Cardiovasc 1996; 11 (3): 137-42.

cendente para coronária esquerda através de enxerto de artéria carótida homóloga em căo, que faleceu poucos minutos após a interrupção do fluxo na coronária. Desde então, diversos autores tentaram a revascularização do miocárdio sem CEC, sem sucesso.

Em 1975, ANKENEY (11), em editorial, revelou ter realizado 200 operaçōes sem o uso da CEC e preconizou que o miocárdio tolerava 20 minutos de isquêmia, enquanto era realizada a anastomose. Outros autores o seguiram relatando suas experiências, como SUZUKI (12), BENETTI et al. $(13,14)$, BUFFOLO et al. $(15,16)$ e ARCHER et al (17).

TRAPP \& BISARIA (18), em 1975, e AKINS (19), em 1983, realizaram revascularizaçăo sem CEC com uso de derivaçǒes de Polietileno e tipo Javid para artéria carótida, respectivamente, com a finalidade de manter o fluxo coronariano. No entanto, estas derivaçōes mostraram-se ser de dificil utilizaçāo, o que reduziu a sua aplicaçăo em larga escala.

A técnica cirúrgica utilizando uma derivaçăo de silicone colocada intraluminal na coronária (DILT) foi descrita por RIVETTI \& GANDRA (20) e RIVETTI (21), permitindo a revascularização sem CEC com manutenção do fluxo intracoronariano.

O presente estudo visa demonstrar que a revascularização do miocárdio sem CEC e com DILT é um método eficaz no tratamento de emergência de pacientes submetidos à ATC sem sucesso.

\section{CASUISTICA E MÉTODOS}

No período de novembro de 1989 a dezembro de 1995 foram realizadas 600 ATC na Santa Casa de São Paulo e Hospital Samaritano, tendo ocorrido insucesso em 18 pacientes portadores de lesōes nas coronárias RIA, CD e Dg, sendo levados a cirurgia de emergência para revascularizaçāo do miocárdio sem auxilio da circulação extracorpórea.

Todos os doentes foram operados, sem o uso da CEC e com auxilio do DILT.

A idade variou de 37 anos a 74 anos, com média de 58,38 anos. O sexo masculino predominou com $12(66,66 \%)$ doentes e $6(33,33 \%)$ do sexo feminino. Todos eram da raça branca.

Dos 18 doentes, apenas 3 apresentavam infarto do miocárdio prévio e nenhum deles havia sido sub. metido a revascularização prévia.

Em $13(72,2 \%)$ doentes a lesăo coronária era única e em $5(27,8 \%)$ havia comprometimento de 2 artérias.

O insucesso caracterizou-se por impossibilida- de de dilataçāo de lesāo critica com angina persistente, dissecção de placa ou oclusāo aguda.

Três $(16,66 \%)$ pacientes apresentavam-se em choque quando levados para o centro cirúrgico, 9 $(50 \%)$ encontravam-se com hipotensāo arterial e 6 $(33,33 \%)$ permaneciam estáveis.

Todos receberam enxerto de veia safena.

O tempo transcorrido do acidente até a realizaqăo da operaçăo foi inferior a 2 horas em $12(66,66 \%)$ doentes e de 2 horas a 12 horas em $6(33,33 \%)$ doentes, sendo estes mantidos com heparina e nitrato via endovenosa.

Como cuidados pré-operatórios, foram realizadas a tricotomia 0 a tipagem sangüinea. Os pacientes foram monitorizados com cateter para pressão arterial média (PAM) e cateter para pressăo venosa central (PVC).

As operaçōes foram realizadas sem a montagem do aparato para CEC e a incisăo cirúrgica foi a mediastinotomia anterior longitudinal transesternal.

A anastomose de veia safena na aorta foi realizada sob pinçamento lateral $e$, ao final, procedemos a heparinizaçāo na dose de $1 \mathrm{mg} / \mathrm{kg}$ de peso.

Isolada a artéria coronária e reparada através de torniquetes de silicone com fio de Prolene 4.0. Aberta a coronária e introduzida o DILT de calibre adequado, restabelecendo-se o fluxo coronariano. A técnica cirúrgica utilizada foi a descrita por RIVETTI (21) e está ilustrada na Figura 1. O DILT, tubo de silicone de diversos calibres, conforme já descrito, é mostrado na Figura 2.

Os cuidados pós-operatórios foram os habituais através de cateteres de PAM, PCV e em átrio esquerdo colocado no intra-operatório.

\section{RESULTADOS}

Foram realizadas 23 anastomoses utilizando-se enxerto de veia safena.

As coronárias revascularizadas foram: RIA, Dg e CD. Foram realizadas 11 pontes de safena para - RIA, 3 pontes para Dg e 9 pontes para CD. Em $5(27,77 \%)$ pacientes foram realizadas 2 pontes de safena por paciente (Tabela 1).

Em 1 caso o RIA apresentava-se intramuscular, sendo realizada anastomose para a $1^{\text {* }}$ diagonal, pois havia lesāo proximalmente a este ramo.

Um paciente foi levado ao centro cirúrgico com cateter de perfusăo para controle de dor persistente.

Um paciente com crise hipertensiva e convulsōes na mesa de hemodinâmica, controladas com 
Pinto A M R, Pochini M, Marinelli I, Campagnucci V P, Gandra S M A, Rivetti L A - Revascularização do miocárdio sem circulação extracorpórea com derivaçăo intraluminal temporária (DILT) na emergència pós angioplastia transluminal coronária (ATC). Rev Bras Cir Cardiovasc 1996; 11 (3): 137-42.

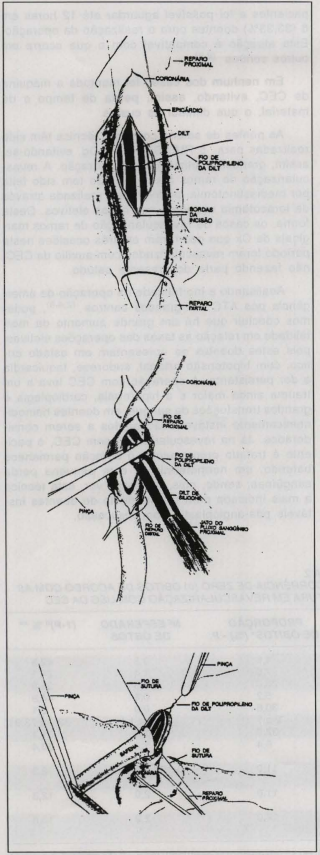

Fig. 1- Técnica cirürgica

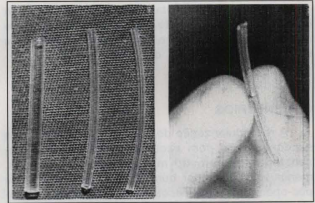

Fig. 2 - DILT

sedaçāo, foi levado para o centro cirúrgico e anestesiado; no pós-operatório, este doente permaneceu com hemiplegia transitória, tendo alta sem seqüelas.

O estudo hemodinâmico revelou hipocinesia de VE em $4(22,22 \%)$ doentes.

O estudo eletrocardiográfico mostrou que 3 $(16,66 \%)$ doentes apresentavam onda q quando chegaram à sala de operaçōes e mantiveram este padrāo no pós-operatório. Quatro $(22,22 \%)$ pacientes apresentavam supradesnivelamento do seguimento ST, que desapareceu após a revascularização. Os $11(61,11 \%)$ restantes apresentaram alteraçōes isquêmicas, havendo melhora no pós-operatório.

Foi realizado estudo estatístico para verificar se a ausência de óbitos da nossa casuística é comparável com a literatura mundial, referente às operaçōes de revascularizaçāo com CEC realizadas na emergência pós ATC complicada.

TABELA 1

CARACTERIZAÇÄO DA CASUISTICA

\begin{tabular}{cc}
\hline N /IDADE /SEXO & REVASCULARIZAÇĀO \\
\hline $1-63 / \mathrm{M}$ & $\mathrm{CD}$ \\
$2-57 / \mathrm{M}$ & $\mathrm{CD}$ \\
$3-74 / \mathrm{F}$ & $\mathrm{DA}$ \\
$4-55 / \mathrm{M}$ & $\mathrm{DA}, \mathrm{IDg}$ \\
$5-63 / \mathrm{F}$ & $\mathrm{DA}, \mathrm{CD}$ \\
$6-46 / \mathrm{M}$ & $\mathrm{CD}$ \\
$7-58 / \mathrm{F}$ & $\mathrm{DA}$ \\
$8-67 / \mathrm{M}$ & $\mathrm{Dg}$ \\
$9-55 / \mathrm{M}$ & $\mathrm{DA}$ \\
$10-55 / \mathrm{M}$ & $\mathrm{CD}$ \\
$11-37 / \mathrm{M}$ & $\mathrm{DA}$ \\
$12-68 / \mathrm{F}$ & $\mathrm{DA}$ \\
$13-48 / \mathrm{M}$ & $\mathrm{DA}, \mathrm{CD}$ \\
$14-52 / \mathrm{M}$ & $\mathrm{CD}, \mathrm{DA}$ \\
$15-65 / \mathrm{M}$ & $\mathrm{DA}$ \\
$16,60 / \mathrm{F}$ & $\mathrm{CD}, \mathrm{Dg}$ \\
$17-62 / \mathrm{F}$ & $\mathrm{DA}$ \\
$18-66 / \mathrm{M}$ & $\mathrm{CD}$ \\
\hline
\end{tabular}


Pinto A M R, Pochini M, Marinelli I, Campagnucci V P, Gandra S M A, Rivetti L A - Revascularizaçăo do miocárdio sem circulaçăo extracorpórea com derivaçăo intraluminal temporária (DILT) na emergência pós angioplastia transluminal coronária (ATC). Rev Bras Cir Cardiovasc 1996; 11 (3): 137-42.

A Tabela 2 apresenta o número esperado de óbitos e a probabilidade de ocorrência zero, de acordo com as proporçōes de óbitos em pacientes submetidos às cirurgias descritas na literatura.

\section{COMENTÁRIOS}

A revascularizaçāo do miocárdio sem CEC vem sendo utilizada com excelentes resultados, apresentando recuperaçāo mais rápida dos doentes, menor custo e menor número de complicações no pós-operatório. Nos casos de insucesso de angio. plastias, verificamos que a revascularização do miocárdio sem CEC tem um papel importante, podendo - paciente ser tratado prontamente, sem necessidade do aparato da CEC (BUFFOLO (22), 1994).

Na Santa Casa de São Paulo, iniciamos a re. alizaçäo de revascularizaçăo sem CEC com DILT em 1983 (RIVETTI \& GANDRA (20)).

Com o início da angioplastia em 1989, pudemos realizar a revascularizaçăo do miocárdio sem CEC com DILT também nos casos de insucessos de ATC.

Os pacientes com dissecçăo de placa, oclusăo aguda ou impossibilidade de dilatação de lesão crítica, que representam as complicaçōes das ATCs realizadas em nosso Serviço, apresentaram fluxo coronariano após a colocaçāo da DILT, a qual é inicialmente colocada na porção proximal e, a seguir, na distal, permitindo que ofluxo se reestabeleça no interior da artéria coronária.

A nossa atuaçăo foi imediata em $12(66,66 \%)$ pacientes e foi possivel aguardar até 12 horas em $6(33,33 \%)$ doentes para a realização da operaçăo. Esta atuaçāo é compativel com o que ocorre em outros centros $(6,9)$.

Em nenhum dos casos foi montada a máquina de CEC, evitando, assim, perda de tempo e de material, o que diminui os custos.

As pontes de safena com esta técnica têm sido realizadas para o RIA, DA, CD e Dg, evitando-se, assim, grandes manipulações do coração. A revas. cularizaçāo de ramos marginais não tem sido feita por mediastinotomia, podendo ser realizada através de toracotomia esquerda nos casos eletivos. Desta forma, os casos de revascularizaçāo de ramos marginais da $\mathrm{Cx}$ que ocorreram em três ocasiōes neste periodo foram revascularizados com auxílio da CEC, não fazendo parte do presente estudo.

Analisando a mortalidade na operaçăo de emergência pós ATC em grandes centros $(2,4-8)$, podemos concluir que há um grande aumento da mortalidade em relação às taxas das operaçōes eletivas, pois estes doentes se apresentam em estado critico, com hipotensão arterial, sudorese, taquicardia e dor persistente. A operaçăo com CEC leva a um trauma ainda maior e a hipotermia, cardioplegia $e$ grandes transfusões de sangue, em doentes hemodinamicamente instáveis são pontos a serem considerados. Já na revascularizaçăo sem CEC, o paciente é tratado prontamente, o coraçăo permanece batendo, em normotermia, e com pequena perda sangüinea, sendo, pois, ao nosso ver, esta técnica a mais indicada para o tratamento de doentes instáveis pós-angioplastia com insucesso.

TABELA 2

NÚMERO ESPERADO DE OBITOS E PROBABILIDADE DE OCORAENCIA DE ZERO (O) ÓBITOS DE ACORDO COM AS PROPORÇOESS DE ÓBITO (\%) DESCRITAS EM LITERATURA EM REVASCULARIZAÇĀOO COM USO DA CEC

\begin{tabular}{|c|c|c|c|c|c|}
\hline AUTORES & LOCAL & $\begin{array}{c}\text { CARACTERISTICA } \\
\text { DO ESTUDO }\end{array}$ & $\begin{array}{c}\text { PROPORÇĀO } \\
\text { DE OBITOS* }(\%) \text { - P }\end{array}$ & $\begin{array}{c}N^{2} \text { ESPERADO } \\
\text { DE OBTOS }\end{array}$ & $(1-P)^{n} \% *$ \\
\hline $\begin{array}{l}\text { Boylan e col. } \\
1995\end{array}$ & Cloveland & $\begin{array}{l}\text { períado de } 80-85 \\
\text { período de } 85-90 \\
\text { pacientes em choque }\end{array}$ & $\begin{array}{r}4,6 \\
7,6 \\
28,3\end{array}$ & $\begin{array}{l}0,8 \\
1,4 \\
0,8\end{array}$ & $\begin{array}{l}42,8 \\
24,1 \\
36,9\end{array}$ \\
\hline $\begin{array}{l}\text { Dallan e col. } \\
1990\end{array}$ & $\begin{array}{l}\text { São Paulo } \\
\text { (InCór) }\end{array}$ & $\begin{array}{l}\text { pacientes estáveis } \\
\text { pacientes graves }\end{array}$ & $\begin{array}{r}5,2 \\
30,8\end{array}$ & $\begin{array}{l}0,8 \\
0,9\end{array}$ & $\begin{array}{r}44,9 \\
3,1\end{array}$ \\
\hline $\begin{array}{l}\text { Carey e col. } \\
1994\end{array}$ & Londres & $\begin{array}{l}\text { pacientes estáveis } \\
\text { pacientes graves }\end{array}$ & $\begin{array}{l}2 \text { a } 7 \\
32,0\end{array}$ & 0,3 a 1,1 & $\begin{array}{c}33,7 \text { a } 73,9 \\
31,4\end{array}$ \\
\hline $\begin{array}{l}\text { Cowley e col. } \\
1984\end{array}$ & Virginia & total de pacientes & 6,4 & 1,2 & 30,4 \\
\hline $\begin{array}{l}\text { Naunheime col. } \\
1989\end{array}$ & St.Louis & total de pacientes & 11,0 & 2,0 & 12,3 \\
\hline $\begin{array}{l}\text { Killen e col. } \\
1984\end{array}$ & Kansas & total de pacientes & 11,0 & 2,0 & 12,3 \\
\hline $\begin{array}{l}\text { Parsonnet e col. } \\
1987\end{array}$ & New Jersey & total de pacientes & 12,0 & 2,2 & 10,0 \\
\hline
\end{tabular}

- número esperado de óbitos $=$ ( $n$.p) sendo $n=18$ (total de pacientes), ou 15 (se pacientes estáveis), ou $n=3$ (se pacientes graves).

** (1-p)n = probabilidade de ocorrerem zero óbitos, em n pacientes, supondo-se a probabilidade de óbito igual a p. 
Pinto A M R, Pochini M, Marinelli I, Campagnucci V P, Gandra S M A, Rivetti L A - Revascularizaçăo do miocárdio sem circulaçăo extracorpórea com derivaçăo intraluminal temporária (DILT) na emergência pós angioplastia transluminal coronária (ATC). Rev Bras Cir Cardiovasc 1996; 11 (3): 137-42.

Pela realizaçăo do eletrocardiograma, verificamos que apenas $3(16,66 \%)$ doentes apresentaram infarto do miocárdio, porcentagem inferior ao que se encontra na literatura (7), o que nos leva a pensar que o bom resultado que obtivemos seja devido a reperfusăo precoce do miocárdio através do uso do DILT.

A permanência na UTI foi de 2,1 dias e a alta hospitalar ocorreu em média de 7,18 dias, demonstrando que a recuperaçăo pós-cirúrgica foi semeIhante aos da operação eletiva.

A mortalidade no presente estudo foi zero. Realizando-se estudo estatístico, verificamos que o número de óbitos esperado em nossa casuística seria um, quando analisamos os resultados dos trabalhos de BOYLAN et al. (9), DALLAN et al. (6), CAREY et al. (2) e COWLEY et al. (7).

No entanto, se considerarmos as proporçōes de óbitos de NAUNHEIM et al. (4), KILLEN et al (5) e PARSONNET et al. ${ }^{(3)}$, verifica-se que seriam espe- rados 2 óbitos dentre os pacientes da Santa Casa, valor este superior ao que realmente ocorreu. Isso leva a pensar que a nāo ocorrência de óbitos neste estudo é um resultado melhor do que o esperado, de acordo com os estudos destes últimos centros.

\section{CONCLUSÕES}

1) Preconizamos o uso da revascularizaçăo do miocárdio sem CEC com DILT pós insucesso de ATC, pois o resultado tem se mostrado superior ao da literatura com uso de CEC.

2) É possível ser utilizada esta técnica cirúrgíca para revascularização de coronárias da face anterior e diafragmática do coraçāo, RIA, $C D$ e Dg.

3) Não é necessária a montagem do aparato da CEC, o que iria aumentar o tempo para atuação cirúrgica e o custo.

\section{RBCCV 44205-301}

Pinto A M R, Pochini M, Marinelli1, Campagnucci VP, Gandra S M A, Rivetti L A - Myocardial revascularization without cardiopuimonary bypass with temporary intraluminal shunt: emergential approachafter transluminal coronary angioplasty. Rev Bras Cir Cardiovasc 1996; 11 (3): 137-42 .

ABSTRACT: From November 1989 through December 1995, eighteen patients underwent emergency coronary artery bypass grafting without cardiopulmonary bypass following percutaneous transluminal coronary angioplasty (PTCA). All patients had lesions in the left anterior descending coronary artery, diagonal coronary artery or right coronary artery, when we could reach without using cardiopulmonary bypass. In all cases we used a temporary intraluminal shunt in order to allow perfusion through the coronary artery and prevent ischemic effects. Three $(16.66 \%)$ patients had myocardial infarction before going to emergency surgery and 4 (22.22\%) patients had important ST segment elevation. The cardiogenic shock was present in $3(16.66 \%)$ patients. The in-hospital mortality was zero. We compared the several authors mortality rates and ours by a statistic analysis.

DESCRIPTORS: Myocardial revascularization, methods. Myocardial revascularization, surgery. Angioplasty. Coronary vessels, surgery. Blood vessels prostheses. Extracorporeal circulation.

\section{REFERÊNCIAS BIBLIOGRÁFICAS}

1 Gruentzig A - Transluminal dilatation of coronary artery stenosis. [Letter], Lancet 1978; 1:263.

2 Carey J A, Davies S W, Balcon R et al. - Emergency surgical revascularization for coronary angioplasty complications. Br Heart $J 1994$; 72: 428-35.

3 Parsonnet V, Fisch D, Gielchinsky I - Emergency operation after angioplasty. I Thorac Cardiovasc Surg 1988; 96: 198-203.

4 Naunheim K S, Fiore A C, Fagan D C et al. - Emergency coronary artery bypass grafting for failed angioplasty: risk factors and outcome. Ann Thorac Surg 1989; 47: 816-23.

5 Killen D A, Hamaker W R, Reed W A - Coronary artery bypass following percutaneous transluminal coronary angioplasty. Ann Thorac Surg 1985; 40: 133-8.

6 Dallan L A, Oliveira S A, Cecchy $H$ et al. Revascularização miocárdica de urgência após complicaçăo de angioplastia transluminal coronária: abordagem cirúrgica atual. Rev Bras Cir Cardiovasc $1990 ; 5: 42-53$. 
Pinto A M R, Pochini M, Marinelli I. Campagnucci V P, Gandra S M A. Rivetti L A - Revascularizaçăo do miocárdio sem circulaçăo extracorpórea com derivaçāo intraluminal temporária (DILT) na emergência pós angioplastia transluminal coronária (ATC). Rev Bras Cir Cardiovasc 1996; 11 (3): 137-42.

7 Cowley M, Dorros G, Kelsey S F, Van Raden M, Detre $\mathrm{K} \mathrm{M}$ - Emergency coronary bypass surgery after coronary angioplasty. The National Heart Lung and Blood Institute's percutaneous transluminal coronary angioplasty registry experience. Am J Cardiol 1984; 53: $22 \mathrm{C}-6 \mathrm{C}$.

8 Ischinger T, Zack P. Aker U - Acute coronary occlusion during balloon angioplasty due to intracoronary thrombus and coronary spasm: a reversible complication. Am Heart $J$ 1984; 107: 1271-5.

9 Boylan M, Lythe B, Taylon P et al, - Have PTCA failures requiring emergent bypass operation changed? Ann Thorac Surg 1995; 59: 283-7.

Carrel A - On the experimental surgery of the theracic aorta heart. Ann Surg 1910; 52: 83-95.

11 Ankeney $\mathrm{JL}$ - To use or not to use the pump-oxygenator in coronary bypass operations. Ann Thorac Surg 1975; 19: 108-9.

12 Suzuki A - Aortocoronary bypass without cardiopulmonary bypass. Jap J Thorac Surg 1977; 25: $629-31$

13 Benetti F J, Rizzardi J L, Aun R, Naselli G, Beltrame R G - Cirurgia coronaria directa con puente de safena sin circulación extracorpórea: experiencia. Rev Arg Cardiol 1985; 53: 225-31.

14 Benetti F J, Naselli G, Wood M, Geffner L - Direct myocardial revascularization without extracorporeal circulation: experience in 700 patients. Chest 1991: 100: $312-6$
15 Buffolo E, Andrade J C S, Succi J E et al. Revascularizaçāo direta do miocárdio sem circulaçăo extracorpórea: descriçăo da técnica e resultados iniciais. Arq Bras Cardiol 1982; 38: 365-73.

16 Buffolo E, Andrade J C S, Succi J E et al. . Revascularizaçăo direta do miocárdio sem circulaçäo extracorpórea: estudo crítico dos resultados em 391 doentes. Rev Bras Cir Cardiovasc 1986; 1: 32-9.

17 Archer R, Ott D A, Parravicini R et al - Coronary artery revascularization without cardiopulmonary bypass. Texas Heart $J$ 1984; 11: 52-7.

18 Trapp W G \& Bisarya A - Placement of coronary artery bypass graft without pump oxygenator. Ann Thorac Surg 1975; 19: 1-9.

19 Akins C W - Reoperation for stenotic saphenous vein bypass grafts without cardiopulmonary bypass. Ann Thorac Surg 1983; 35: 201-6.

20 Rivetti L A \& Gandra S M A - Revascularizaçăo cirúrgica do miocárdio sem auxilio da circulaçāo extracorpórea, com derivaçăo interna temporária. Rev Soc Cardiol Estado de Săo Paulo 1991; 1: 65-71.

21 Rivetti L A - Revascularização do miocárdio sem circulaçåo extracorpórea com derivação intraluminal temporária [Tese.Doutorado]. Săo Paulo: Faculdade de Ciências Médicas da Santa Casa de Misericórdia de Săo Paulo, 1992.180p.

Buffolo E - Revascularizaçăo miocárdica sem circulação extracorpórea: resultados cirúrgicos em 1090 pacientes. Arq Bras Cardiol 1994; 62: 149-53. 